\title{
Reflets
}

Revue ontaroise d'intervention sociale et communautaire

\section{Représentations des TIC en milieu professionnel : réflexions sur le changement}

\section{Julie Boissonneault}

Volume 9, numéro 2, automne 2003

Travail et mieux-être

URI : https://id.erudit.org/iderudit/011098ar

DOI : https://doi.org/10.7202/011098ar

Aller au sommaire du numéro

Éditeur(s)

Reflets : Revue ontaroise d'intervention sociale et communautaire

ISSN

1203-4576 (imprimé)

1712-8498 (numérique)

Découvrir la revue

Citer cet article

Boissonneault, J. (2003). Représentations des TIC en milieu professionnel :

réflexions sur le changement. Reflets, 9(2), 207-221.

https://doi.org/10.7202/011098ar

Tous droits réservés (C) Reflets : Revue ontaroise d'intervention sociale et communautaire, 2002
Ce document est protégé par la loi sur le droit d'auteur. L'utilisation des services d'Érudit (y compris la reproduction) est assujettie à sa politique d'utilisation que vous pouvez consulter en ligne.

https://apropos.erudit.org/fr/usagers/politique-dutilisation/ 


\section{Représentations des TIC en milieu professionnel : réflexions sur le changement}

\section{Julie Boissonneault}

Professeure, Département d'études françaises et de traduction, Université Laurentienne, Sudbury

La représentation du monde n'est plus adaptée au contexte, mais à la mondialisation du savoir, à la virtualisation de nos perceptions. Nos yeux et nos oreilles ne nous servent, dans ce monde-là, qu'à percevoir les signifiants minuscules qui véhiculent et engendrent une représentation majuscule (Cyrulnik 2001: 252).

Depuis la venue des technologies de l'information et de la communication (TIC), temps et espace ne sont plus les mêmes. L'implication de leur redéfinition nous a tous amenés à revoir nos façons de faire et à repenser nos repères spatiotemporels. La présence des TIC - inforoute, courriel, cellulaire, vidéoconférence, entre autres - s'est fait sentir dans toutes les sphères sociales, y compris dans le marché du travail. Dans ce numéro portant sur les pratiques professionnelles, il est tout à propos de se poser des questions sur comment nous conjuguons avec les TIC dans nos milieux professionnels respectifs, puisqu'il se pourrait fort bien que nous partagions les mêmes préoccupations. Après tout, les TIC sont présentes dans plusieurs situations personnelles et professionnelles; nous les avons tous intégrées, à des degrés différents, dans nos façons de faire, dans nos façons de penser, et ce, peu importe la profession exercée. 
Cet article propose d'abord un regard sur le concept des représentations sociales comme mesure de la façon dont on conjugue avec le changement, puis un regard sur son interprétation dans le cadre d'une profession donnée: celle des professeurs d'université. Le lecteur aura le loisir d'inférer à partir de cette situation particulière ce qui se rapporte à son expérience professionnelle. Les représentations dont il sera question portent sur l'intégration des TIC dans la pratique professorale en analysant les défis tant passés qu'à venir sur la médiatisation de l'enseignement universitaire. Elles ont été recueillies dans le contexte de la formation à distance et l'analyse qu'il en est fait s'inscrit dans une recherche menée en milieu universitaire en 2001.

\section{Pourquoi parler de représentations?}

Parler de représentations, c'est en fait parler d'images et de valeurs collectives. L'étude des représentations permet de mieux comprendre les changements qui ont cours et comment les joueurs s'y adaptent. Elle permet tout particulièrement de cerner l'appropriation d'un nouvel élément et sa restructuration dans les façons de penser et de faire. En l'occurrence, les TIC sont ce nouvel élément. Je pars de la prémisse qu'elles ne laissent personne indifférent: pour les uns, c'est une panacée, pour les autres, un fléau.

La force des représentations réside en ce qu'elles vont au-delà des spécificités individuelles en faisant éclore le discours collectif, c'est-à-dire tout ce qui se recoupe dans les discours personnels. Parler de représentations, c'est ainsi déplacer l'angle d'études du plan perceptuel (forcément individuel) vers un plan représentatif de la collectivité. Il s'en dégage un schème de pensée qui illustre et qui explique la dynamique du groupe. Bien que tout individu faisant partie d'un groupe donné puisse partager ou non certaines composantes de ce schème, l'étude des représentations sociales a comme postulat que la représentation que se fait un groupe est plus que la somme des perceptions individuelles. 
Depuis quelques années, il est d'ailleurs de plus en plus question de représentations (socio) professionnelles pour chercher à comprendre l'adaptation de regroupements d'individus au sein d'une même profession et les changements qui s'y opèrent. Un nombre toujours croissant d'études se penchent sur de telles représentations dans des disciplines ou des champs particuliers, notamment les sciences de la santé (Lebrun 2001) et le monde de l'éducation (Karsenti et al. 2001). La visée des représentations sociales est de cerner comment un collectif quelconque s'approprie sa réalité à la lumière d'un ou de plusieurs changements, et comment il la structure ou la restructure en fonction de ce qui est.

Deux grands angles permettent de circonscrire les représentations sociales: par objectivation et ancrage, d'une part, par noyau central et élément périphériques, d'autre part. Dans le premier cas, le savoir collectif (processus d'objectivation) est confronté au savoir individuel (processus d'ancrage) (Doise et al. 1992; Guimelli 1994, 1995). L'objectivation permet de tabler sur ce que tous partagent, puis de faire intervenir le nouvel élément en l'ancrant à ce que l'on connait déjà. Dans le second cas, il est question de délimiter le noyau central de la question à l'étude et d'en identifier les éléments périphériques (Abric 1994). Le nouvel élément peut ainsi être évalué selon qu'il se retrouve soit au cœur même de ce qui est, soit en périphérie. Le changement ou la situation est évidemment tout autre dans l'un ou l'autre des scénarios. Si le nouvel élément se retrouve au cœur même de la situation, il restructure complètement ce qui était. Par contre, s'il n'est qu'en périphérie, il s'ajoute à ce qui est. Qu'en est-il alors des TIC?

C'est la question qui est posée? Mais à prime abord, que donne l'étude des représentations sociales dans un contexte universitaire? Premièrement, les représentations sont symptomatiques des changements en ce qu'elles pilotent les comportements: exprimer la représentation que l'on se fait d'une situation conduit à la compréhension que l'on en dégage. Deuxièmement, le choix d'étudier les représentations dans un contexte universitaire reconnaît à l'université le rôle qu'elle joue dans le transfert technologique, celui qu'elle joue dans le développement des compétences nécessaires pour utiliser et pour organiser ces 
technologies et les organiser, puis son rôle de chef de fil au chapitre de la recherche, de l'avancement scientifique et de la formation des chercheurs (Tuijnman 1996). Finalement, le fait d'ancrer l'étude dans le cadre de la formation à distance - formation hautement tributaire des médias — permet d'aller au cœur de la situation, là où les questions sur la présence, sur l'intégration et sur la pertinence des TIC se posent de façon réelle et concrète. Il est alors plus aisé de chercher à comprendre la place qu'occupent les TIC auprès de professeurs les ayant utilisés: comment se les représentent-ils dans leur pratique d'enseignement et d'évaluation de l'apprentissage et comment cette représentation a-t-elle joué sur l'utilisation qu'ils en ont fait? Bref, quelle réflexion tiennentils à l'égard des TIC et comment cette réflexion se traduit-elle dans leur pratique professionnelle?

\section{Méthodologie}

Pour répondre à ces questions, j'ai choisi de procéder par une instrumentation d'ordre qualitatif. Une dizaine de professeurs toutes disciplines confondues ${ }^{1}$ — se sont prêtés à des entretiens semi dirigés. L'échantillon était conforme au critère d'homogénéisation en ce que tous les répondants provenaient du même établissement: milieu universitaire bimodal, c'est-à-dire où se pratique à la fois l'enseignement en face à face et la formation à distance. Il était aussi conforme au critère de diversification de la saturation empirique par la diversification disciplinaire (5 disciplines) et le partage homme-femme ( 5 hommes, 5 femmes). Le but premier était de vérifier l'hypothèse selon laquelle l'intégration des TIC bouscule les façons de faire dans la pratique professionnelle des professeurs et les amène à réfléchir à leur rôle, puis de dégager ce qui, dans les discours, se prêtait à une imagerie collective. Les participants partageaient certaines caractéristiques en ce qu'ils enseignaient tous au niveau universitaire, ils étaient tous expérimentés (ayant en moyenne 18 années d'expérience à leur actif) et ils avaient tous fait appel à des médias interactifs dans le 
cadre de la formation à distance, notamment la vidéoconférence, l'audioconférence, l'inforoute ou une combinaison des trois.

Dans le cadre des entretiens, les répondants ont d'abord exposé ce que représentent l'enseignement et l'apprentissage universitaires pour eux en précisant leurs attentes, leur formation à enseigner et leur cheminement de carrière. En faisant intervenir l'élément des TIC, ils ont ensuite dressé le portrait de leur expérience en formation à distance et de leur expérience avec les TIC — quel que soit celles retenues - resituant ces dernières dans leur pratique et permettant de mieux comprendre où ils se situaient à cet égard en tant que professeur.

Les discours tenus par les répondants ont été enregistrés pour en faciliter la transcription et le verbatim de l'entretien leur a été soumis aux fins d'approbation. Les données ainsi recueillies ont aussi été corroborées par une analyse documentaire (plans de cours et outils d'évaluation ou activités d'apprentissage) et par la tenue d'un journal de bord, ce qui a permis de compléter l'information. Étaient ainsi juxtaposés le dit et le faire permettant la triangulation des données.

L'analyse de contenu s'est faite en deux temps: a priori, la saisie des unités de sens pour une première classification, puis, a posteriori, une catégorisation thématique. C'est cette dernière étape qui fera ressortir le discours collectif.En guise de vérification supplémentaire, la codification, la catégorisation et la thématisation ont été soumises à une évaluation inter juges de deux personnes externes qui en ont vérifié la cohérence et la logique en y apportant un éclairage différent, dans certains cas.

\section{Que se dégage-t-il des données?}

Il est difficile, voire impossible, de rendre justice à la richesse des données recueillies en quelques pages. Ce qui suit ne fait état d'aucune spécificité individuelle mais représente les grandes lignes des discours telles qu'elles se rapportent à la question posée au 
début: l'apport des représentations sur l'intégration des TIC dans le milieu professoral.

Trois des questions de l'entretien tombaient sous le primat de l'enseignement universitaire, permettant aux répondants d'exprimer d'abord leur représentation de ce signifie être professeur, leur cheminement pédagogique ou comment ils en étaient venus à exercer leur profession et les transformations défis, nouveautés, réflexions - qu'ils ont noté en cours de route et de préciser le contexte dans lequel ils travaillent. Une autre question les amenait ensuite à préciser leurs attentes quant à la visée de l'enseignement:l'appropriation et la construction du savoir disciplinaire et professionnel, visée par ailleurs partagée par tous et réitérée à maintes reprises.

L'apprentissage des connaissances c'est la base mais ensuite à partir de ces connaissances-là, qu'est-ce qu'on en fait? Alors il faut aller plus loin que la simple acquisition de connaissances. [...] Il faut aller dans la remise en question des connaissances (04-FR).

L'importance accordée au développement de la pensée critique, tant du point de vue du but de l'enseignement que de celui de l'apprentissage, est constante dans tous les discours recueillis. À ceci, les répondants ajoutent que l'enseignement et l'apprentissage, somme toute, se font essentiellement par échanges avec autrui. La constance de la pensée critique sera confirmée dans les propos subséquents et fait ressortir l'importance qu'accordent les répondants à tout ce qui entoure l'interaction, quelle soit médiatisée par les TIC ou dans le face à face.

L'université devrait former à la réflexion, à la pensée critique, à la citoyenneté. Ensuite elle devrait former des professionnels compétents dans les différents domaines (06-SS).

Trois autres questions d'entretien ont permis de dégager l'image qu'ils se font de la formation à distance qui, on s'en souviendra, est le contexte dans lequel se fait la collecte des données. L'image qu'ils s'en font et celle qu'ils estiment être 
véhiculée par les étudiants éclairent leurs discours en faisant ressortir les valeurs qu'ils y attribuent et les valeurs véhiculées par le milieu. Dans l'un ou l'autre des cas, les valeurs sont partagées. Il n'y a entente que sur l'utilité et sur la pertinence de palier la distance.

Il y a beaucoup de profs qui pensent, je pense, que donner des cours à distance c'est facile parce qu'on fait rien. On n'est pas là trois heures dans la classe à rien faire. Je trouve au contraire que ça demande beaucoup de temps parce que au niveau de la correction ça en demande beaucoup plus. Parce que comme c'est le seul feedback que les étudiants ont ou presque,je pense que ce feedback-là est extrêmement important (04-FR).

La pratique de la formation à distance où l'on fait appel à des médias interactifs ouvre la porte à une réflexion sur l'usage proposé et réel des TIC en milieu professionnel, en permettant de suivre le cheminement des individus dans l'intégration qu'ils en ont fait à titre de professeurs. La formation à distance, comme mode d'enseignement et d'apprentissage, fait réfléchir, il va sans dire. Que s'en dégage-t-il? Certains des repères spatiotemporels sur lesquels les répondants s'appuyaient n'y sont plus. Il revient alors au professeur de s'adapter. Cette adaptation peut être plus ou moins réussie selon la motivation à ce faire.

La formation à distance, c'est un concept extraordinaire, mais c'est pas juste donner des cours non plus. C'est de trouver des moyens pour rejoindre les gens, pour que les gens nous rejoignent, pour répondre encore à leurs besoins. [...] Alors la distance pour moi, c'est la flexibilité des gens à avoir de la formation. Et puis la flexibilité va dans les deux sens. C'est pas juste l'étudiante qui vient à nous. Il faut aller à elle. [...] Il va falloir que nous autres aussi on soit flexible, puis que... la formation à distance c'est rejoindre les gens, la flexibilité (01-SI).

Mais au-delà de la formation à distance, les répondants ont également à surmonter un défi de taille: intégrer les TIC à leur pratique afin d'augmenter les possibilités d'interaction, laquelle, 
si on en juge aux propos qu'ils tiennent, est au cour de l'enseignement et de l'apprentissage. La pertinence des TIC n'est donc pas remise en question; elle est toutefois relativisée à la lumière de la visée collective qu'est la communication.

Quatre questions portant sur l'expérience des répondants face aux TIC permettent de savoir, d'une part, s'ils ont reçu une formation quelconque pour intégrer les TIC et pour les utiliser dans leur enseignement et, le cas échéant, la nature de cette formation. L'entretien leur permet également d'exprimer et de faire valoir les compétences qu'ils jugent nécessaires pour enseigner avec les TIC.

C'est la livraison qui est souvent difficile sur le plan expérientiel. [...] C'est un mode d'éducation qui demande, à mon avis, une disponibilité de la part du prof dans le sens que ma philosophie à moi c'est ce que je dis aux étudiants: "Si vous avez une question, vous me téléphonez. Si vous avez un ordinateur, vous m'envoyez un courriel. " Parce que le courriel pour moi c'est plus rapide que le téléphone, parce que souvent je suis dans des rencontres (05-SS).

La poursuite de l'entretien nous amène au cœur de l'intégration des TIC en pratique professionnelle: quel usage en ont-ils fait? C'est à ce chapitre que revient l'importance de l'interaction que permettent les médias, importance déjà soulignée dans leur représentation de l'enseignement. C'est d'ailleurs l'importance de l'interaction qui, à leurs dires, a motivé leur choix de faire appel aux TIC. Les mots-clés " échanges, discussions, questions et participation" reviennent constamment dans les discours tenus. Les explications qu'ils donnent quant aux activités et aux stratégies mises en oœuvre pour ce faire se présentent sous deux angles particuliers: d'abord, l'utilisation particulière des médias avec tout ce qu'ils sous-tendent de potentiel et de contraintes, puis les stratégies d'évaluation, lesquelles décrivent comment les répondants voient cette composante. 
Moi je pense que c'est beaucoup plus difficile d'enseigner à distance que d'enseigner sur le campus. [...] Quand on a les étudiants en face à face, c'est plus facile de voir leurs difficultés. C'est dans leur face. Quand les étudiants ne sont pas en face à face, on n'a pas le pouls tangible de l'individu. On ne sait pas où il est. On ne sait pas où il se situe. On n'a aucune idée de ses problèmes, sauf par les travaux quelquefois, et même là c'est difficile (04-FR).

Et puis la difficulté qu'on rencontre - le défi pour mettre ça plus positif - c'est comment faire vivre aux étudiantes à distance la richesse que je peux vivre sur campus avec les étudiantes parce qu'elles sont là. Mais au plan de la qualité, pour moi, le cours c'est le même, la même base, la même théorie (05-SS).

Peu importe le média choisi, il est évident que tous les répondants ont choisi ou ont accepté de faire appel à l'inforoute, à l'audioconférence ou à la vidéoconférence avec l'intention première d'interagir plus fréquemment avec les étudiants, de leur permettre d'échanger entre eux et, ce faisant, de palier la distance.

\section{Représentations sur les TIC en milieu professoral}

L'analyse des discours tenus par les répondants - discours dans lesquels ils se resituaient, ils se questionnaient, ils discutaient - a permis de juxtaposer un ensemble d'images individuelles de ce que signifie être professeur, de ce qu'est enseigner à distance et de la place des TIC dans le milieu universitaire. Â partir des images personnelles, il a été possible de circonscrire ce qui relevait de la personne et ce qui était partagé entre tous. C'est ainsi que s'est tissée la représentation collective. Tel était d'ailleurs l'objet de la recherche. 
Pour reprendre le concept des représentations, il importe de cerner ce qui est au cœur de l'enseignement et de l'apprentissage universitaires, et ce, dans le but de situer la présence des TIC dans un schème de pensée collectif. Bien que chacun des répondants y soit allé selon ses spécificités, tous partagent une visée commune face à ce que signifie être professeur: la quête du savoir sur le plan personnel et l'appropriation de ce savoir par les étudiants. Ce savoir est bien plus que factuel, il est remis en question, il est pensée critique. Les répondants attendent d'eux-mêmes ce qu'ils attendent des étudiants. Ce savoir s'approprie et se construit par la communication interpersonnelle à laquelle les médias interactifs peuvent contribuer. À ce chapitre, les TIC sont aux yeux de tous un outil de communication, par lequel il est possible d'échanger dans le but de construire le savoir. Les TIC ne sont donc pas le noyau central de l'activité d'enseignement et d'apprentissage, mais un élément périphérique qui y contribue.

Mais comme il s'agit d'un nouvel élément, qu'advient-il alors lorsqu'on ne maîtrise pas cet élément? Les répondants détiennent selon leur champ d'expertise, le savoir disciplinaire et le savoir professionnel qu'ils ont acquis depuis de nombreuses années en enseignant en face à face. Mais peu d'entre eux détiennent le savoir qui se rapporte à la compréhension des médias interactifs et la compétence à les utiliser à bon escient en situation d'échanges, non entre pairs, mais à titre professoral.

Je pense qu'on ne s'exprime pas de la même manière au niveau de la formulation, des explications. Il faut être beaucoup plus spécifique et [...] il faut presque deviner ce que les gens n'ont pas dit parce qu'ils peuvent pas le dire comme en classe [...] Il faut avoir cette espèce de feeling et se dire: "Bon si j'étais de l'autre côté, pourquoi je n'aurais pas compris?" Alors que quand tu es en classe tu peux discuter et ça marche pas comme ça. Çaje l'ai découvert avec l'audioconférence (10-CO).

Tous les répondants se sont ainsi retrouvés, à un moment ou un autre, en situation d'apprentissage face aux «nouvelles» technologies avec lesquelles ils transigeaient, ce qui les a rendu 
vulnérables. Les TIC sont alors, dans ce cadre, un élément perturbateur. Il importe de se souvenir, comme j'en faisais état au tout début, que les TIC bousculent les repères spatiotemporels: temps et espace ne sont plus ce à quoi ils étaient habitués. Par exemple, l'audioconférence et la vidéoconférence sont des médias en temps réel, dits synchrones; dans le premier cas, le visuel n'y est pas, dans le second, la polémique résulte souvent de la croyance que face à face et vidéoconférence sont interchangeables, ce qui n'est pas le cas. C'est le repère spatial qui differe ici. Quant à l'inforoute, les scénarios varient selon l'usage qu'on en fait: le courriel et le babillard, bien qu'ils accélèrent les échanges, sont des médias à temps différé (dits asynchrones). On croit souvent à tort que l'envoi d'un message est signe d'immédiateté: les attentes des professeurs et des étudiants peuvent ainsi être tronquées. Le bavardoir (communément appelé clavardage et chat) permet des interactions en temps réel, lesquelles se conjuguent souvent dans un espace-temps délimité. Ces deux activités interactives par inforoute - courriel / babillard en différé et bavardoir ou clavardage en réel — font appel à des échanges écrits, contrairement aux conférences auditives ou visuelles qui sollicitent l'expression orale. Le média ou la combinaison de modalités interactives peut ainsi faire appel à des stratégies d'enseignement autres que celles valorisées et habituelles dans le face à face. Malgré leur expérience, les répondants ont ainsi été appelés à intégrer un outil sur lequel ils exerçaient peu de contrôle en raison d'un manque de préparation et de formation. Il n'y a donc pas lieu d'être surpris si l'outil occupe alors une place plus grande que ce qui devrait lui être dévolu.

Or, avant de se prêter à l'exercice de l'intégration des technologies interactives, le scénario ne semblait pas, aux yeux des répondants, très difficile. Il ne s'agissait que d'une technicalité. L'expérience fait surgir des questions tant sur la fonctionnalité que sur ce qui se rapporte au cœur même de l'enseignement l'établissement de rapports et d'interactions réelles. Les interactions qui découlent de ces rapports se présentent en deux temps: en temps réel et en temps différé. Ils redéfinissent aussi l'espace. Doiton être surpris outre mesure par le fait que les nouveaux médias 
interactifs suscitent un questionnement sur des éléments tenus pour acquis au chapitre professionnel.

Alors comment les répondants s'y sont-ils pris pour intégrer les TIC à leur pratique professionnelle? D'emblée, la majorité (huit des dix répondants) allèguent l'avoir fait de façon autodidacte, par un apprentissage sur le tas et au fur et à mesure que le besoin se faisait sentir. Les deux autres avaient antérieurement reçu une formation pédagogique, leur permettant de faire appel à des compétences transférables pour conjuguer avec les TIC. Chose certaine, aucun répondant ne reconnaît avoir reçu, en milieu professionnel et dans le cadre de ses fonctions, une formation qui répondait pleinement à ses préoccupations, qui apaisait ses craintes et qui correspondait à ses besoins. Les formations reçues sont davantage, pour ne pas dire exclusivement, d'ordre technique: elles portent sur le fonctionnement de la technologie. L'apprentissage des TIC s'est donc fait souvent seul et avec peu d'appui: leurs discours traduisent fort bien leurs inquiétudes et leurs réflexions à cet égard. Or, c'est d'une formation «techno pédagogique», ancrée selon les besoins de la pratique professorale, qu'ils disent avoir besoin: encadrement et appui pour établir des interactions, pour mieux comprendre les contraintes et le potentiel des médias et pour retraduire leur expérience en stratégies médiatiques qui tiennent compte des différences spatiotemporelles.

Ce questionnement amène, par la force des choses, un renforcement de la représentation de ce que signifie être professeur pour les répondants. C'est ainsi que surgit la centralité des représentations. Les répondants font valoir l'importance de l'interaction comme gage de la construction du savoir, interaction qui en elle-même suscite la réflexion critique. Or, réflexion critique et remise en question sont les deux versants d'un même schème. Par ricochet, les TIC placent les professeurs à la fois comme initiateurs et instigateurs de la remise en question, et récepteurs de cette même remise en question. 


\section{Quel est l'apport des représentations au chapitre professionnel?}

Les représentations qui se sont dessinées dans le cadre des entretiens ont permis de repositionner la question de la centralisation: qu'estce qu'être professeur? La réponse resituera les TIC en milieu universitaire. L'image collective qui se dégage du discours des répondants est le fruit de leurs observations, de leurs expériences et de leurs questionnements par lesquels ils jettent un regard critique sur leurs façons de faire. Émerge ainsi leur posture à l'égard de la façon dont ils se représentent: spécialistes dans leur champ disciplinaire, expérimentés dans le savoir enseigner, mais nouveaux au jeu de la médiatisation. Ce dernier élément leur a permis de laisser émerger une nouvelle posture dans leur rapport au savoir. Tous aspirent à l'appropriation du savoir, appropriation parallèle bien que différente de celle que recherchent les étudiants.

Leurs représentations font valoir que la relation pédagogique et l'appropriation du savoir ne sont pas à la remorque de l'infrastructure technologique. L'interaction (l'échange avec autrui) est primée et recherchée, bien que le milieu forme à l'interactivité (le machinique). Par objectivation, on fait état de ce qui est acquis (l'expérience, le vécu) auquel on greffe, par ancrage, le nouvel élément que sont les TIC. Les répondants ne se représentent pas cet élément comme étant le noyau central: bien au contraire. Mais tel n'est pas toujours le discours véhiculé dans le milieu professoral, voire dans les milieux professionnels.

L'importance d'une réelle formation correspondant aux besoins professionnelles est on ne peut plus claire. Mais cette formation doit tenir compte des différences spatiotemporelles qu'exigent les TIC. Les répondants l'ont bien vu en juxtaposant leur expérience antérieure à celle des TIC. La formation technique ne fait que remplacer un média par un autre (le connu par le nouveau), entraînant ainsi soit une mésadaptation du nouvel élément, soit une adaptation douloureuse et peu valorisée. 
Les professeurs l'ont bien cerné: l'échange est au cœur de l'enseignement et de l'apprentissage. Les TIC en formation à distance répondent à ce besoin d'interaction et rehaussent les possibilités de communication. Mais les communications interactives que permettent les médias ne sont pas les mêmes que celles valorisées dans la formation traditionnelle, et ces différences ne sont pas toujours reconnues à leur juste valeur par le milieu professionnel.

Dégager les représentations en milieu professionnel a permis, dans un contexte de changement, de remettre le cap sur ce qui était important à la profession. Il est alors beaucoup plus aisé pour l'institution ou l'organisme, à la lumière des constats représentatifs, d'envisager et de prévoir un changement planifié et approprié. Il le sera tout autant pour le professionnel qui cherche à comprendre l'ambiguité du changement qu'il vit. Il lui est alors possible de voir la « représentation majuscule » dont parle Cyrulnik dans son ouvrage L'ensorcellement du monde (2001).

\section{Bibliographie}

Abric, J. C. (sous la dir. de) (1994). Pratiques sociales et représentations, Paris, Presses universitaires de France.

Cartier, M. (1997). Le nouveau monde des infostructures, (s.1.), Fides.

Cartier, M. (2001). «Les inforoutes et l'éducation, mythes et réalités», dans M. Kaszap, D. Jeffrey, et G. Lemire, éd., Exploration d'Internet, recherches en éducation et rôles des professionnels de l'enseignement, Québec, Les Presses de l'Université Laval / Paris, L'Harmattan, 9-59.

Cyrulnik, B. (2001). L'ensorcellement du monde, Paris, Odile Jacob.

Doise, W., A. Clemence et F. Lorenzi-Cioldi (1992). Représentations sociales et analyses de données, Grenoble, Presses universitaires de Grenoble.

Doise, W. et A. Palmonari (sous la dir. de) (1986). L'étude des représentations sociales, Neuchâtel, Delachaux et Niestlé.

Gumelli, C. (1994). Structures et transformations des représentations sociales, Neuchâtel, Delachaux et Niestlé.

Guimelli, C. (1995). «L'étude des représentations sociales», Psychologie Française, vol. 40, no 4, 367-374.

Karsenti, T. et F. Larose (sous la dir. de) (2001). Les TIC... au coeur des pédagogies universitaires. Diversité des enjeux pédagogiques et administratifs, Sainte-Foy (Québec), Presses de l'Université du Québec.

Karsenti, T., L. Savoie-Zajc et F. Larose (2001, Printemps). «Les futurs enseignants confrontés aux TIC: changements dans l'attitude, la motivation et les pratiques pédagogiques», Éducation et francophonie. Revue scientifique virtuelle, vol. 29, no 10. Page consultée le 4 juin 2001. [En ligne]. Adresse URL: http://www.acelf.ca/revue/XXIX-1/articles/03-Karsenti.html 
Lebrun, M. (sous la dir. de) (2001). Les représentations sociales. Des méthodes de recherche aux problèmes de société, Outremont (Québec), Les Éditions Logiques.

Lévy, P. (1997). L'intelligence collective. Pour une anthropologie du cyberspace, Paris,

La Découverte.

Savoie-Zajc, L. (1993). Les modèles de changement planifié en éducation, Montréal, Les Éditions Logiques.

Tuijnman, A.C. (sous la dir. de) (1996). International Encyclopedia of Adult Education and Training (2 ed.), New York, Elsevier Science Inc.

\section{Note}

1. Les participants provenaient des disciplines suivantes: sciences infirmières, service social, français, commerce et administration, sciences pures et appliquées. 